\title{
Erratum
}

Due to an unfortunate oversight, one of the authors' corrections was not carried out. The list of authors and the affiliations should have been printed as follows:

\section{Inheritance of mitochondrial and chloroplast genome markers in backcrosses of Chlamydomonas eugametos $\times$ Chlamydomonas moewusii hybrids}

Robert W. Lee ${ }^{1}$, Bonny Langille ${ }^{1}$, Claude Lemieux ${ }^{2}$, and Poppo H. Boer ${ }^{3 *}$

Departments of ${ }^{1}$ Biology and ${ }^{3}$ Biochemistry, Dalhousie University, Halifax, Nova Scotia B3H 4J1, Canada

${ }^{2}$ Département de biochimie (Sciences), Université Laval, Québec, Québec G1K 7P4, Canada 